\title{
Drop-charge Correlations for Polydisperse Electrostatically Atomized Liquid Sprays
}

\author{
Martin C. Y. Wong and John S. Shrimpton \\ Thermofluids Section, Department of Mechanical Engineering \\ Imperial College of Science, Technology and Medicine \\ London, SW7 2BX, England
}

\begin{abstract}
In many applications liquid sprays are atomized using electrostatic methods, and typically these spray plumes containing drops that have a range of diameters. To understand and predict the dynamics of polydisperse electrically charged spray plumes, knowledge of how the electrical charge is distributed amongst the drops is required. This has been achieved by post-processing phase Doppler anemometry data for two electrostatically atomized liquid sprays and fitting the drop diametercharge correlation to an assumed relationship of form $q=A D^{n}$. Here $q$ and $D$ are drop charge and diameter and $n$ and $A$ are empirical constants that describe the correlation. Values of $n$ and $A$ were calculated to be 2.1 to 2.9 and $5.8 \times 10^{-5}$ for a spray of specific charge $1.8 \mathrm{C} / \mathrm{m}^{3}$ and 2.1 to 3.2 and its value of $A$ is $2.5 \times 10^{-4}$ for a spray of specific charge $1.2 \mathrm{C} / \mathrm{m}^{3}$. It was found that the mean drop charge, for all drop diameters, for both data-sets, was almost always less than the drop Rayleigh limit. This latter fact gives confidence in the procedure used since no restriction was placed on this parameter during the processing. We also estimate the distribution of drop charge about the mean value and as a function of diameter and suggest that small drops possess higher rms charge levels.
\end{abstract}

Index Terms - Electric charge, drop, Rayleigh Limit.

\section{INTRODUCTION}

$\mathrm{T}$ HE electrostatic atomization of conducting and semi-conducting liquids is now a valuable part of many industrial processes; examples being paint and crop spraying [1], non impact printing [2], monosize metal particle production [3] and pollution abatement [4]. Much research has been completed to investigate the stability of charged drops but studies have tended to concentrate on a single drops and not the stochastic nature of drop charge distributions. Early workers employed a force balance principle in Millikan type apparatus to calculate drop diameter and charge independently of one another to investigate the single drop charge limit, the Rayleigh limit [5].

$$
q_{m}=\frac{4 \pi}{\sqrt{2}}\left(\epsilon_{o} \sigma_{T}\right)^{1 / 2} D_{m}^{3 / 2}
$$

here $q_{m}$ and $D_{m}$ denote the drop charge and drop diameter of the $m$ th size class of a drop diameter probability density function (PDF). $\epsilon_{o}$ and $\sigma_{T}$ represent the permittivity of the continuum in which the drop resides and the liquid surface tension coefficient, respectively.

Doyle et al. [6] investigated aniline and water drops in the range $200 \geq D \geq D 60 \mu \mathrm{m}$. Pfeifer and Hendricks [7]

Manuscript received on 16 July 2002, in final form 8 December 2003 correlated the data of Krohn [8] and Hendricks [9] for small drops of conducting liquids, Schweizer and Hanson [10] investigated $n$-octanol drops in the range $40 \geq D \geq 15$ $\mu \mathrm{m}$ and Abbas and Latham [11] examined the stability of water, aniline and toluene drops of size $200 \geq D \geq 30 \mu \mathrm{m}$. These early studies, within the limits of their experimental accuracy, confirmed the Rayleigh limit. They also show that for a given drop diameter a range of drop charges are present. The early studies did not however investigate the correlation between probable drop charge and drop diameter. They are of little use to researchers wishing to predict the dynamics of polydisperse charged sprays using computational methods, since there is no reason why the charge of a drop should be a defined function of the Rayleigh limit. The Rayleigh limit simply informs us of the maximum charge the drop may hold, and nothing about the probability that it has any given charge below that limit. Also the Rayleigh limit provides no information concerning how much charge one drop possesses, relative to another drop of the same diameter, nor one of a different diameter.

Since the instability of liquid jets and sheets that creates liquid drops is non-linear, we would expect the joint PDF (JPDF) of drop charge and drop diameter to also be non-linear. However no information on the drop charge diameter JPDF of any atomisation devices has been pub- 
lished as far as the authors are aware, and it is this omission that this contribution seeks to address.

Later work (Gomez and Tang [12-14], Talfin et al., [15] and Hirabayashi [16]) belongs to a second subject area, that of electrospray characterisation [17, 18]. This literature is growing at a rate approaching 1000 articles a year [19] and covers theory, scaling, laws of operation, effects of liquid, geometry and operating parameters and novel applications. The characteristics of an electrospray typically include two separate monomodal highly constrained drop diameter PDFs, one of which is populated by very small drops. A 'cone-jet' is required to create this monosized electrospray and this requires a liquid of specific properties, in particular the electrical conductivity of the liquid should exceed $10^{-5} \mathrm{~S} / \mathrm{m}$. For quasi-mono size charged drop generation, a cone-jet is normally employed, and since flow rate, drop diameter and spray current may either be measured or obtained by semi-empirical scaling laws [20], drop charge may be estimated with some degree of confidence.

The same cannot be said for polydisperse charged sprays however, such as those produced by charged injection atomizers [21, 22] and electrostatically assisted spinning cup atomizers such as those found in the car body spray applications [23]. Here it is not known how the charge imparted to the spray is distributed (a) amongst the different size classes and (b) within a single class. This knowledge is required if we wish to be able to predict the dynamics of the spray and estimate parameters such as the distribution of deposition flux to a surface, a parameter important in the quality control of coated surfaces.

The key experimental problem is that although it is possible to capture a single drop from a charged spray, and measure its charge and mass with a range of techniques [24], it would only be possible at the spray edge, and, in statistical terms, only a few drops could be measured. Furthermore these drops would on average, be the most highly charged and not representative of the spray as a whole. Therefore in this work we seek to develop a method of defining mean and root mean square (rms) drop charge by post-processing method of data obtained from phase Doppler anemometry (PDA) measurements [25], where data on the velocities and diameters of individual drop are available. The concept was first applied by Schwar et al. [26] using Schlieren interferometry to calculate the mobility velocities of charged particles, thus we extend this method to polydisperse sprays by making use of the JPDF of diameter and velocity available through phase Doppler anemometry instrumentation.

The method of Schwar et al. [26] is extended in three respects. Firstly the Schlieren method has a large sample volume $\left(\sim 5 \times 10^{-2} \mathrm{~cm}^{3}\right)$ as opposed to the PDA sample volume of $\sim 6 \times 10^{-6} \mathrm{~cm}^{3}$, permitting much improved spatial resolution. Secondly, the Schlieren method only recorded velocity, or, for a known electric field, particle

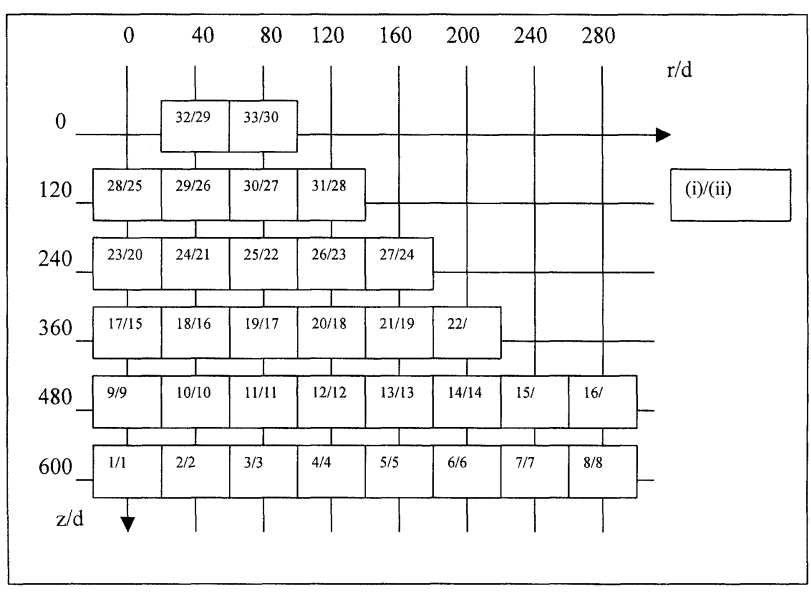

Figure 1. Schematic diagram of PDA measuring grids. The measuring points are numbered in ascending order. Number on the left is measuring point for data-set (i) and numbers on the right is the measuring point for data-set (ii).

mobility. It was not possible to separate drop charge from drop diameter, as in the proposed method. With PDA drop velocity and drop size are measured independently and simultaneously. Thirdly, PDA is a single particle counting device, whereas the Schlieren method of Schwar et al. [26] integrates the signal from all particles in the collection volume. Therefore great care must be taken to obtain single particle information with the latter method.

\section{PROCEDURE}

The data, discussed in detail elsewhere [25], was obtained using a 2D PDA system, therefore axial and radial velocities and diameters of individual drops are available for the 2D measurement plane defined in Figure 1, where the nozzle is positioned at the coordinate origin and the spray is coaxial with $z$ axis. Here axial $(z)$ and radial $(r)$ ordinates have been normalized by the orifice diameter, and data-sets (i) and (ii) are defined in Table 1. $\bar{u}$ is the mean liquid velocity through the orifice of the atomizer calculated from volumetric flow Bombenkova "Measurement orifice area. $Q_{V}$ is the volumetric spray specific charge calculated from the quotient of the electric current carried by the spray and the volumetric liquid flow rate and $N$ is the total number of drops measured by the PDA in the data-set. The numbers in Figure 1 at the $(z, r)$ coordinates refer to measurement locations in the spray plume where data was obtained. The two data-sets are generated by the same atomizer, of constant orifice diameter but are different in two key respects. The first is the flow rate, as implied by Table 1 from the average velocity of the liquid

Table 1. Properties of datasets (i) and (ii).

\begin{tabular}{l|cccc}
\hline & $d(\mu \mathrm{m})$ & $u(\mathrm{~m} / \mathrm{s})$ & $Q_{v}\left(\mathrm{C} / \mathrm{m}^{3}\right)$ & $\mathrm{N}$ \\
\hline Dataset (i) & $250 \mu \mathrm{m}$ & 10 & 1.2 & 14650 \\
Dataset (ii) & $250 \mu \mathrm{m}$ & 34 & 1.8 & 40700 \\
\hline
\end{tabular}


jet emerging from the atomizer. The second is the spray specific charge, and it is noted that although the specific charge is only $50 \%$ more for data-set (ii), in absolute terms there is much more charge present in the spray plume, because spray specific charge is used. It is known that these spray plume characteristics affect both primary atomization, and spray dispersal rates, as discussed more fully elsewhere [25].

The number of drops recorded per measurement location in general decreased with increasing radial displacement, ranging from up to $10^{4}$ drops at the centerline to up to $10^{2}$ drops at the edge of the spray. With reference to the results of [25], it is clear that mean diameter reduces with increasing $r / d$ and this suggests that drop specific charge increases with reduction in diameter. However it is also apparent that most drop size classes are populated at most radial displacements and this suggests that there exists a joint probability density function of drop charge and diameter, and it is this we seek to quantify for the two data sets defined above.

We assume that the drop charge of the $m$ th drop size class varies with diameter such that

$$
q_{m}=A D_{m}^{n}
$$

where $A$ and $n$ are to be determined. With this general form, for $n \sim 1.5$, we may introduce the Rayleigh Limit, by comparison with (1), $A=8 \pi\left(\epsilon_{0} \sigma_{T}\right)^{1 / 2}$ and, for hydrocarbon oils, where $\sigma_{T} \sim 0.02 \mathrm{~N} / \mathrm{m}, A \sim 10^{-5} \mathrm{C} / \mathrm{m}^{3 / 2}$. Similarly, for $n \sim 2$ we have the possibility of defining a drop governed by a surface field emission limit, commonly encountered when drops or particles are charged by ion bombardment [27]. For perfectly conducting liquids, where the surface of the liquid volume maintains a constant voltage $\phi$, then $\phi=q_{1} / D_{1}=q_{2} / D_{2}$ and $n \equiv 1$ by definition, and we may take this as an absolute lower limit of the value of $n$. For a perfectly insulating liquid, providing the surface charge is mobile and if a drop could divide very slowly, $n \equiv 2$, however we cannot take this as an upper limit since it is very unlikely that surface charge on insulating liquids can redistribute itself faster than the surface forms through primary atomisation.

Certain assumptions are made in order to investigate the relationship between the drop charge and the droplet diameter. Firstly, we assume that the spray system has reached steady state, including the electric field which although varying in space is steady in time. Secondly, we assume that the axial and radial momentum equations may be decoupled so that velocities and electric fields in the $z$ and $r$ direction are considered separately. Thirdly we assume that the components of radial drag and coulomb repulsion are balanced and the radial acceleration of the spray droplets is zero. This will apply most accurately to the smallest drop size classes, but also to regions where radial acceleration is small. Predominantly this applies away from the spray axis and at the spray edge. Since there is no correlation between radial velocity and radial electric field on the spray axis, we must ignore these positions.

The experiment was carried out under room temperature and pressure. Due to the difficulty in measuring velocity of the surrounding gas, for instance we cannot simply take the velocity of the smallest size class as characteristic of the gas, the velocity of the gas phase is assumed to be zero. Therefore, for zero radial acceleration, the force balance on the drop in the $r$ direction is given by

$$
F_{D}=F_{E}
$$

The drag force is defined,

$$
F_{D}=\frac{1}{2} C_{D} \rho_{g} \pi \nu^{2}\left(\frac{D}{2}\right)^{2}
$$

where the drop drag coefficient $C_{D}$ is a function of the drop Reynolds number, $\operatorname{Re}=D \nu_{r} \rho_{g} / \mu_{g}$. The relative velocity, $\nu_{r}$, between the drop and the continuum is taken to be the drop velocity as noted above. $\rho_{g}$ and $\mu_{g}$ represent the continuum density and dynamic viscosity. Since our data showed $\operatorname{Re}>1$ we use the drag coefficient definition of Putnam [28], a function of drop Reynolds number

$$
C_{D}=\frac{24}{\operatorname{Re}}\left(1+\frac{(\mathrm{Re})^{2 / 3}}{6}\right)
$$

with the radial electric force, given

$$
F_{E}=q E_{r}
$$

Combining equations (2), (4), (5) and (6) into (2) provides the following equation in terms of our unknown $n$ and $A$ and known $D$ and $v$-component of the velocity vector.

$$
\ln \left\{3 \mu_{g} \nu\left[1+\frac{1}{6}\left(\frac{\rho_{g} D \nu}{\mu_{g}}\right)^{2 / 3}\right]\right\}=(n-1) \ln D+\ln \left(\frac{A E_{r}}{\pi}\right)
$$

Strictly this applies for a single particle, at a point in space. However we assume that, over a measurement duration, this applies to all particles passing through a specific point in space, which we take to be the PDA measurement points.

It should be noted that there are two assumptions in equation (7). There first is the charge relationship between charge and diameter, equation (1). The second is that all drops at a measurement point have the same value of $n$. The constancy of $A$ is addressed later, but it should be noted that, at this stage, there is no assumption made regarding $\mathrm{A}$. If both of these assumptions are valid, then plotting the LHS of equation (7), $\ln \left\{3 \mu_{g} \nu[1+\right.$ $\left.\left.(1 / 6)\left(\rho_{g} D \nu / \mu_{g}\right)^{2 / 3}\right]\right\}$ (denoted $\ln [f(\nu)]$ hereafter), against $\ln (D)$ should produce a straight line of gradient $(n-1)$, and an intercept of $\ln \left(A E i_{\mathrm{r}} / \pi\right)$. The graphical presentations that follow show that this is indeed the case. It should be 
noted that the values of $n$ used for later calculations were obtained using a least squares method and not obtained from visual fits.

\section{RESULTS}

Both data-sets were processed initially to find the distribution of $n$ with $z / d$ and $r / d$. At points along the centre line of the spray $\left(r / d_{0}=0\right)$, there is no radial electric field but turbulence is present. As a result, the radial velocity for each measuring point along the centre has a zero mean, and there is no valid correlation possible for $n$. In addition no correlation of $n$ was found for small $z / d_{0}$, for all $r / d_{0}$. This is because the droplet trajectories are predominantly axial in this region since the spray is being attracted back onto the earthed atomiser body (for further information on this atomization technique refer to [29-31]). Making use of equation (7), we show in Figures $2 a$ and $2 b$ how $\ln (f(v))$ varies with $\ln (D)$ for constant $z / d_{0}$ for data-sets (i) and (ii), where the $z, r$ data co-ordinates may

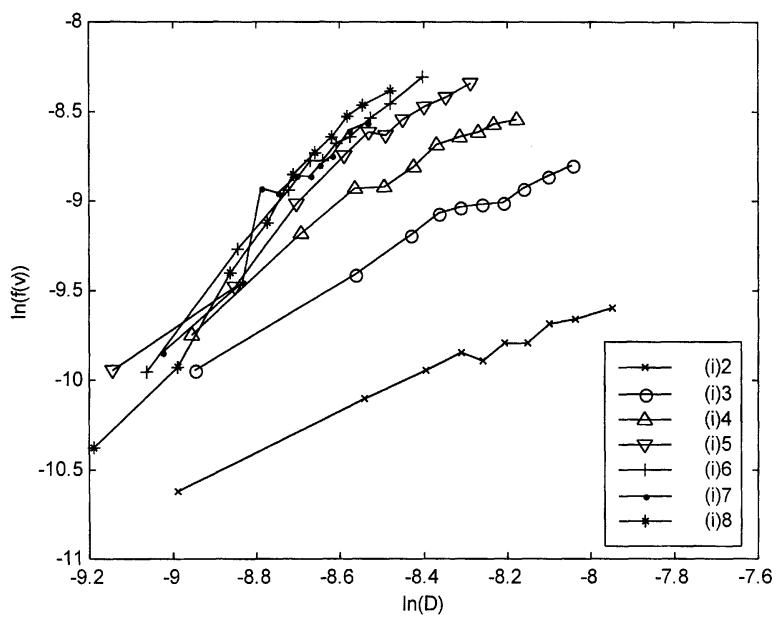

(a)

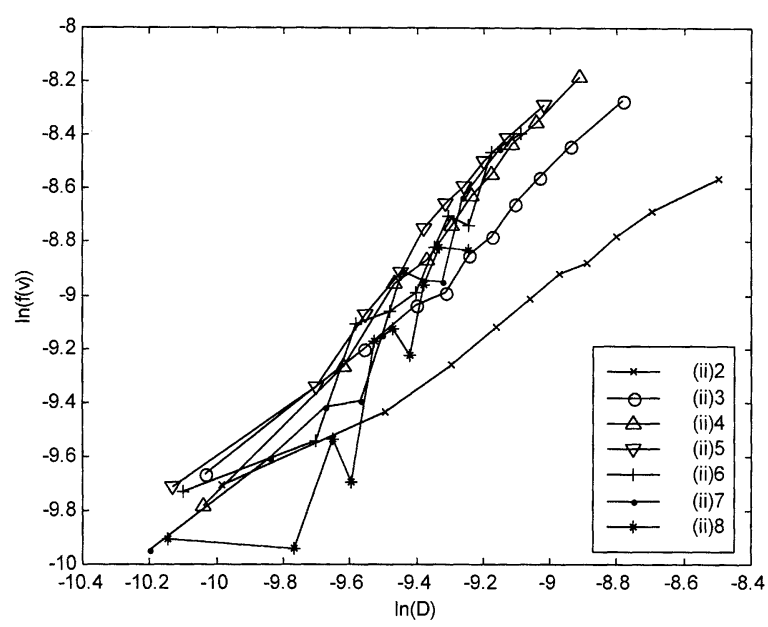

(b) be referenced from Figure 1. The linearity of the correlations is encouraging and oscillations occur primarily where the quantity of data points is small, and not due to any fundamental reason we can illicit, thus we can claim that equation (2) is probably a good estimate of actual drop charge to drop diameter relationship. A clear pattern is evident in that the gradient increases with $r / d_{0}$ with the largest drops showing the most variation. This behaviour is found for both data sets, over the 'core' data range, which excludes data at small $r / d_{0}$, on the spray axis and at small $z / d_{0}$, near to the injector. We also observe that some difficulties arise at the outer edges of the spray, but this is due to a paucity of data rather than a failing of the model, and is discussed in more detail below.

The gradient behaviour of both data-sets, over all valid points from the data processed is shown in Figures 3a and $3 \mathrm{~b}$. Both show the truncations of the valid regions of the correlations clearly, with the region of low data rates clearly defined by the sharp gradient change originating at

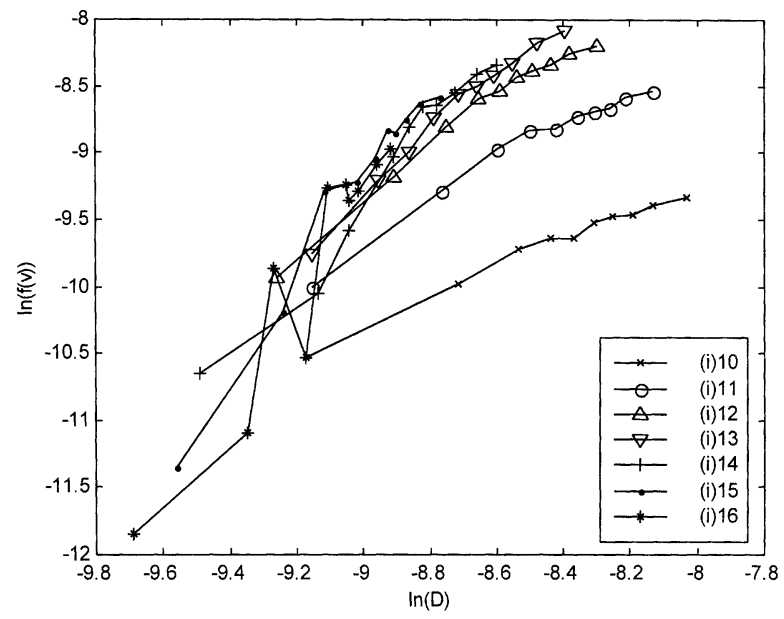

(c)

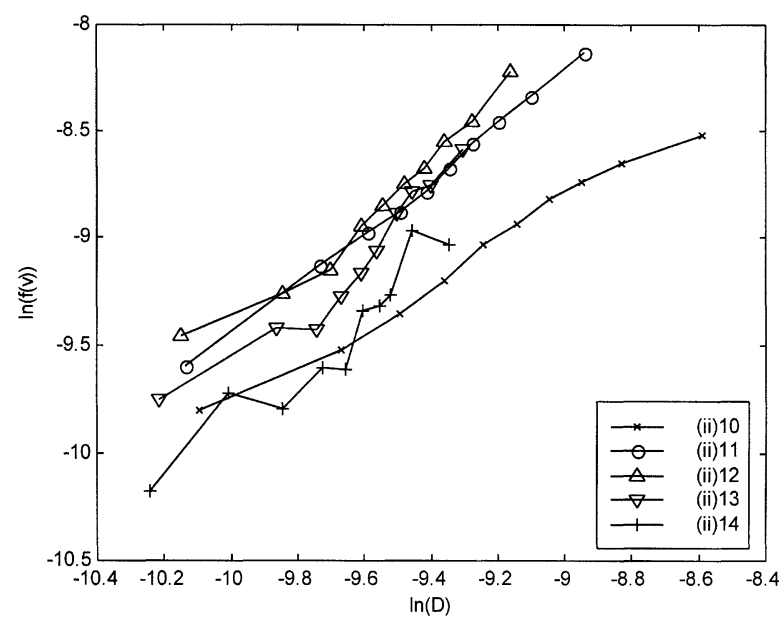

(d)

Figure 2. $\ln (f(\nu))$ versus $\ln (D)$. a, data-set (i) at $z / d=600$ for $280 \geq r / d 40 ; B$, data-set (ii) at $z / d=600$ for $280 \geq r / d 40$; c, data-set (i) at $z / d=480$ for $280 \geq r / d \geq 40 ; d$, data-set (ii) at $z / d=480$ for $200 \geq r / d \geq 40$. 


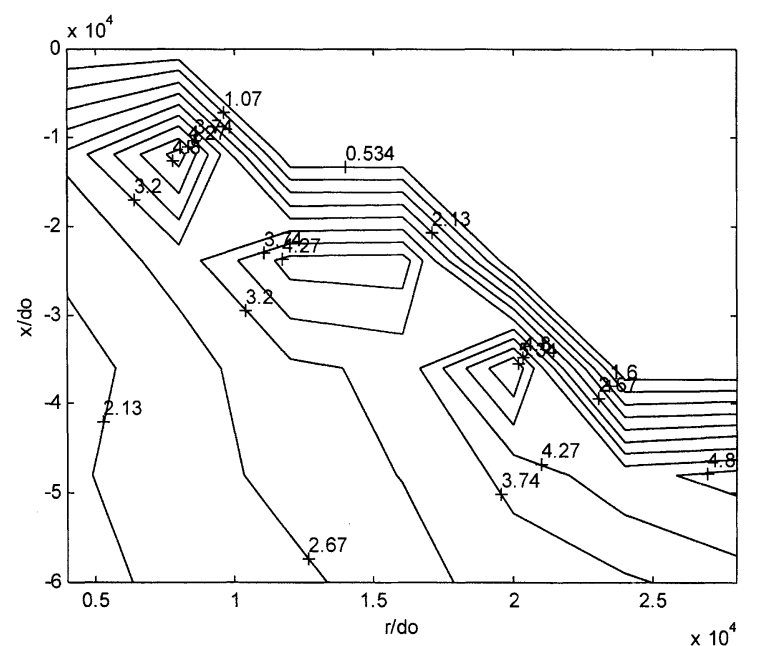

(a)

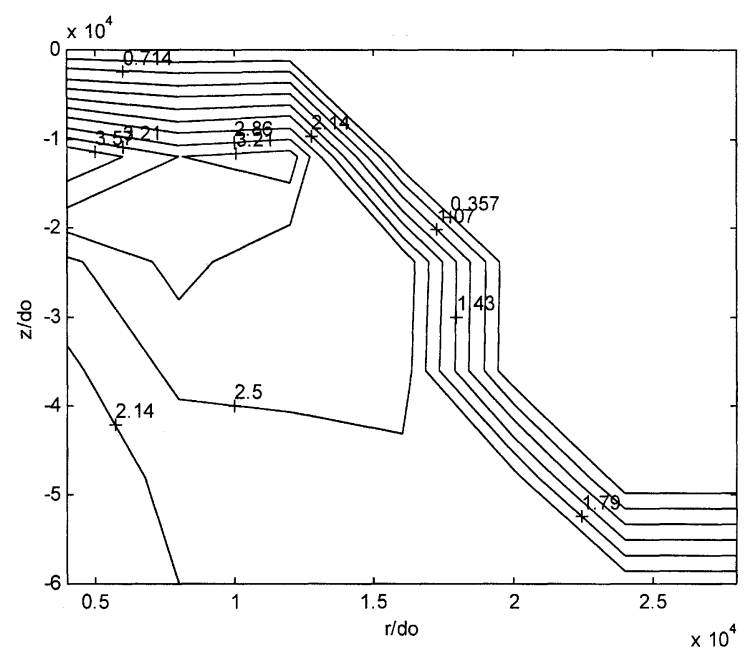

(b)

Figure 3. Spatial distribution of $n$. a, data-set (i); b, data-set (ii).

$(z, r) \sim(0,0)$. For data-set (i), in Figure 3a, the values of $n$ at the edge of the spray are generally higher while the rest of the spray has similar values to data-set (ii) but with less consistency, ranging from 2.1 to 3.2 and the difference is in part due to lower sample numbers for data-set (i). Figure $3 \mathrm{~b}$ shows that the values of $n$ for data-set (ii) throughout the spray is reasonably constant and have the value of 2.14 to 2.85 in most regions except at smaller $z / d_{0}$. The reason for this has already been outlined above.

From the analysis thus far we have values of $n$ for each measurement point, for both data-sets, and now the remaining steps of the procedure are described to obtain estimates of individual drop charges for individual drops. First we take all the drops present at each measurement point and calculate relative drop charges $\left(q^{*}\right)$, using the value of $n$ at each measurement point, e.g. for the $p$ th drop of all drops at a measurement point

$$
q_{p}^{*}=D_{p}^{n}
$$

We now have a JPDF of relative drop charge versus drop diameter over all measurement points, which we assume to be the entire spray. To obtain an absolute JDPF we must estimate the constant $A$ of equation (2).

To obtain an estimate of $A$ we divide the sum the individual (relative) drop charges over all measured drops, by the sum of the individual known drop volumes $(V)$, again over all measured drops to obtain a relative mean specific charge

$$
Q_{V}^{*}=\frac{\sum_{p} q_{p}^{*}}{\sum_{p} V_{p}}
$$

We know the actual volumetric charge from experimental measurements, as listed in Table 1, and it is a simple matter to estimate $A$ by comparing the relative and actual volumetric spray specific charges, $Q_{V}^{*}$ and $Q_{V}$. Since equation (2) holds for all drops,

$$
A=\frac{\pi Q_{V} \sum_{p} D_{p}^{3}}{6 \sum_{p} D_{p}^{n}}
$$

In equation (9) the summation index $p$ applies to all drops in the data-set. The exponent $n$, as discussed above, is constant for all drops at a given measurement location, but varies between measurement locations. $Q_{V}$ is the actual volumetric spray specific charge as listed in Table 1. Note that we only need consider the drops measured, since we are aware $[32,33]$ that the PDA technique, a single particle counter, does not measure absolute flux quantities such as mass flux and concentration accurately. The technique, with perhaps the exception of very small particle sizes $(D \sim 5 \mu \mathrm{m})$, records all particles with equal probability, thus relative measures such as the shape of the drop diameter PDF may be considered accurate.

Using equation (9), we define unique charges on individual drops through calculated values of ' $A$ ' of $2.4 \times 10^{-4}$, and $5.8 \times 10^{-5} \mathrm{C} / \mathrm{m}^{-3 / 2}$, for data-sets (i) and (ii) respectively. We considered the effect of the exceptional values of $n$ at the edges of the sprays and near the nozzle of the spray on the calculated values of ' $A$ ', and also removing the effects of all measurement points with less than 100 drops. With these modifications only the third significant figure of the ' $A$ ' constants changed from both data sets, and this gives us some confidence in our estimate in terms of robustness.

Now we have values for ' $n$ ' for each data point and ' $A$ ' for each data-set, we re-process all drops for all measurement locations, for each data-set to find the mean and standard deviation of drop charge as a function of size class, for each data-set. The result of this operation is shown in Figures 4a and 4b, for data-sets (i) and (ii) respectively. Each data point represents a single drop, the 


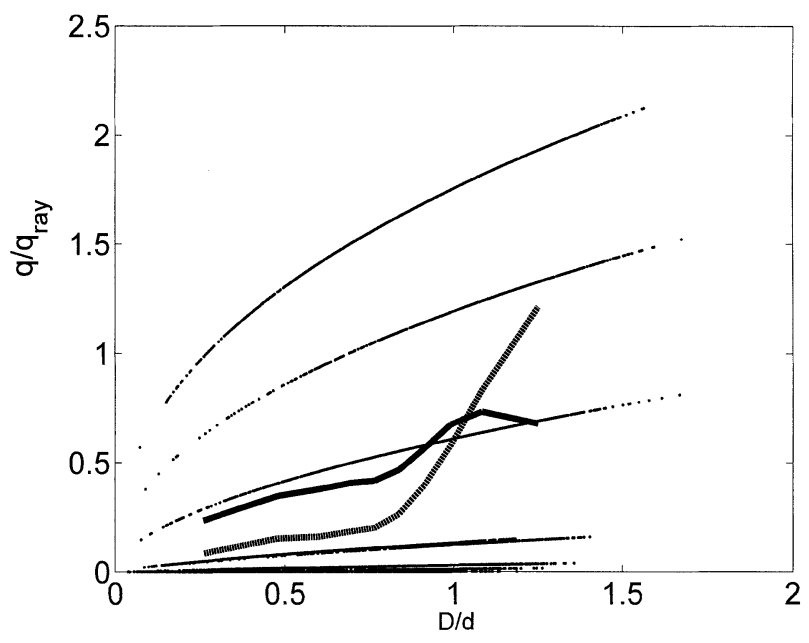

(a)

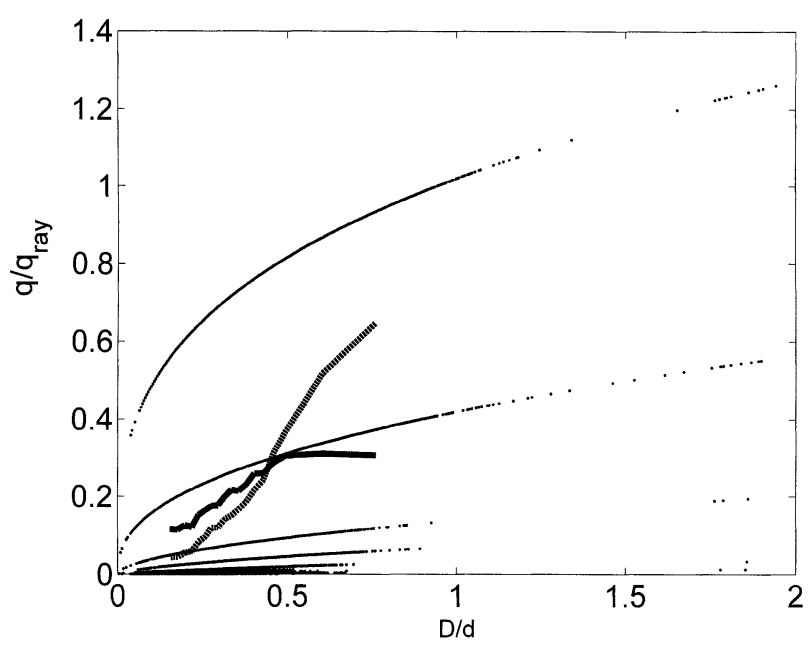

(b)

Figure 4. Plots of normalised individual (points) mean (dashed lines) and rms (solid lines) drop charge versus normalised diameter. a, data-set (i); b, data-set (ii).

dashed lines represent the means, the solid lines the standard deviations about the means, and the dash-dot lines the mean volumetric specific drop charge. The drop diameter is normalised by the orifice diameter, and from Table $1, d=250 \mu \mathrm{m}$. The drop charge, mean charge and rms charge are normalised by the Rayleigh charge defined by equation (1). The specific charge is normalised by the overall (measured) spray specific charge, as defined in Table 1 for each data-set. The mean, rms and specific charge correlations have been truncated either end of the diameter range where numerical oscillations dominate due to low sample number.

Most importantly, and not immediately apparent from Figure 4, is that the majority of drops are predicted to possess very small charges for both data-sets, and in terms of number, most drops lie below the mean charge. Therefore it is relatively few but highly charged drops that pull the mean value up. This is also why the standard deviation of charge lies above the mean, and suggests some form of PDF description may be required to give accurate estimates of drop charge-diameter distribution. The shape of the charge rms is also interesting because it suggests that the calculation of rms charge is not swamped by gas phase turbulence. A typical rms velocity versus diameter results from PDA data would normally show the drop rms rising and approaching the gas rms levels for the smallest drops, falling in the middle of the diameter range and rising again for the largest drops, due to low sample numbers. For the charge rms we accept that a similar reason may be responsible for the larger drops, but for $D / d<1$ for Figure $4 \mathrm{~b}$ and $D / d<0.6$ for Figure $4 \mathrm{~b}$, we are confident we are seeing a true charge rms. However we simply do not have enough data from either set to investigate the shape of the distribution.

There are clear differences in the shape of the correlations between the two data-sets, with data-set (ii) exhibiting a uni-modal character whereas in data-set (ii) there is a clear distinction between the large and the small ends of the drop diameter range. It is suggested that, because the injection velocity for data-set (ii) is much larger, primary break-up is much faster and the charge does not have time to influence the atomization behaviour, such as the formation of ligaments normal to the jet axis, as photographs show elsewhere [22]. This reasoning is supported but the evidence of relatively uniform drop mean specific charge for all drop diameters for data-set (ii), i.e. there is no segregation of drop charge during primary atomisation. This is not the case for data-set (i), which has the slower mean velocity, and the reverse is suggested, that the high charge density containing regions of liquid that 'bud off' from the main jet and form the small drop component of the bimodal drop charge distribution.

Lastly, we point out that for nearly all mean charges of both data-sets, the drop charge to Rayleigh Limit charge ratio is less than 1 , which gives encouragement that the methodology is correct. It is re-iterated that no explicit limit was imposed during our post-processing and gives confidence in our method. The numbers simply come out of the charge conservation calculation and the assumption that the drops recorded by the PDA have the measured spray charge density. We therefore feel that this method does have potential, and is a logical extension of the mobility analogy first proposed by Schwar et al. [26], making use of the JPDF of diameter and velocity, available through PDA instrumentation.

\section{CONCLUSIONS}

A methodology has been explored to define how charge is ascribed to drops of varying sizes within a charged spray, generated by a charge injection atomizer. The processed data from Shrimpton and Yule [25] shows that the values of $n$ in $q_{d, m}=A D^{n}$ range from 
2.1 to 2.8 for a charged kerosene spray with specific charge of $1.8 \mathrm{C} / \mathrm{m}^{3}$ and from 2.1 to 3.2 for the same but different specific charge of $1.2 \mathrm{C} / \mathrm{m}^{3}$. Generally the value of $n$ is constant but there are some extreme cases at the edge of the spray and near the nozzle where $n$ tends to be higher. This is due to lack of measurements at the spray edge and recirculation to the earthed orifice near the nozzle. The value of $A$ is then worked out to be $5.8 \times 10^{-5}$ for the spray with specific charge of $1.8 \mathrm{C} / \mathrm{m}^{3}$ and $2.4 \times 10^{-4}$ for the spray with specific charge of $1.2 \mathrm{C} / \mathrm{m}^{3}$ and further investigation shows that the exceptional values of $n$ have little effect in calculating the value of $A$. Globally the method predicts drop charges less than the Rayleigh Limit for nearly all drops and that small drops are charged to a smaller fraction of the Rayleigh limit than their large cousins but that their distribution of charge about the mean is much wider. Finally, it is accepted that the standard deviations for the smaller size classes are probably overestimated due to the presence of gas turbulence, but it is difficult to estimate the magnitude of the contribution.

\section{REFERENCES}

[1] A. J. Kelly and I. Simmons, "Electrostatic Spraying of Oil Based Agricultural Products", Pesticides and Application Systems, Vol. 5, L. D. Spicer and T. M. Keneko (Eds.), ASTM STP915, American Society for Testing and Materials, Philadelphia, USA, pp. 56-64, 1986.

[2] T. Agui, and M. Nakajima, "Drop Formation Characteristics of Electrostatic Ink jets using a Water Base Ink", IEEE Trans. Electron Devices, Vol. 24, pp. 262-266, 1977.

[3] J. Perel, J. F. Mahoney, B. E. Kalensher, and R. Mehrabian, Advances in Metal Processing, J. J. Burke, R. Mehrabian and V. Weiss (Eds.), Plenum Publishing, New York, pp. 79-89, 1981.

[4] C. N. Ahmed, and W. Balachandran, "Suppression of Smoke Particles using Electrostatic Spraying", ICLASS-91, Gaithersburg, USA, Paper 89, 1991.

[5] Lord Rayleigh, "On the Equilibrium of Liquid Conducting Masses Charged with Electricity", Phil. Mag. 5th series, Vol. 14. pp. $184-185,1882$.

[6] A. Doyle, Read, D. R. Moffett and B. Vonnegut, "Behaviour of Evaporating Electrically Charged Droplets", J. Colloid Sci. Vol. 19. pp. $136-143,1964$.

[7] R. Pfeifer and C. D. Hendricks, "Charge-to-Mass Relationships for Electrohydrodynamically Sprayed Liquid Droplets", Phys. Fluids., Vol. 10, pp. 2149-2154, 1967.

[8] V. E. Krohn, "Progress in Astronautics and Rocketry", D. B. Langmuir, E. Stuhlinger and J. M. Sellen Jr., eds., Academic Press, Vol. 5, pp. 105-110, 1961.

[9] C. D. Hendricks, Proc. 2nd Symposium on Advanced Propulsion Concepts, V. C. Coates and W. Langois, eds., Vol. I, pp. 169, 1959.

[10] J. Schweizer and D. Hanson, "Stability Limit of Charged Drops", J. Colloid Interface Sci., Vol. 35, pp. 417-423, 1971.

[11] M. Abbas and J. Latham, "The Instability of Evaporating Charged Drops", J. Fluid Mech., Vol. 30, part 4, pp. 663-670, 1967.

[12] A. Gomez, and K. Tang, "Charge and Fission of Droplets in Electrostatic Sprays", Phys. Fluids, Vol. 6, pp. 404-414, 1994.

[13] A. Gomez and K. Tang, "Generation by Electrospray of
Monodisperse Water Droplets for Targeted Drug Delivery by Inhalation", J. Aerosol Sci., Vol. 25, pp. 1237-1249, 1994.

[14] A. Gomez and K. Tang, "On the Structure of an Electrostatic Spray of Monodisperse Droplets", Phys. Fluids, pp. 2317-2332, 1994.

[15] D. Taflin, T. Ward and E. Davis, "Electrified Droplet Fission and the Rayleigh limit", Langmuir, Vol. 5, pp. 376-384, 1989.

[16] A. Hirabayashi, "Evaporation of Charged Fine Droplets", Int. J. of Mass Spec. Ion Processes, Vol.175, pp. 241-245, 1998.

[17] M. Cloupeau and B. Prunet-Foch, "Electrostatic Spraying of Liquids : Main Functioning Modes", J. Electrostatics, Vol. 25, pp. $165,1990$.

[18] M. Cloupeau, and B. Prunet-Foch, "Electrohydrodynamic Spraying Functioning Modes: a Critical Review", J. Aerosol Sci., Vol. 25, pp. 1021-1036, 1994.

[19] F. De La Mora and I. G. Loscertales, "The Current Transmitted through an Electrified Conical Meniscus", J. Fluid Mech., Vol. 260, pp. 155-184, 1994.

[20] A. M. Gañan-Calvo, J. Dávia and A. Barrero, "Current and Droplet Size in the Electrospraying of Liquids", J. Aerosol Sci., Vol. 28, pp. 249-275, 1997.

[21] A. J. Kelly, "The Electrostatic Atomisation of Hydrocarbons", J. Inst. Energy, pp. 312-320, 1984.

[22] J. S. Shrimpton and A. J. Yule, "Atomisation, Combustion and Control of Charged Hydrocarbon Sprays", Atomisation and Sprays, Vol.11, pp. 1-32,2001.

[23] Q. Ye, T. Steigleder, A. Scheibe and J. Domnick, "Numerical simulation of the electrostatic Powder Coating Process with a Corona Gun", J. Electrostatics, Vol. 54, pp. 189-205, 2002.

[24] R. C. Brown, "Tutorial Review: Simultaneous Measurement of Particle Size and Particle Charge", J. Aerosol Sci., Vol. 28, pp. 1373-1391, 1997.

[25] J. S. Shrimpton and A. J. Yule, "Characterisation of Charged Hydrocarbon Sprays for Application in Combustion Systems", Experiments in Fluids, Vol. 26, pp. 315-323, 1999.

[26] M. J. R. Schwar, K. C. Thong and F. J. Weinburg, "Measuring the Mobility of Charged Aerosols by Schlieren Interferometry of Doppler-Shifted Light", J. Phys D: Appl. Phys., Vol. 3, pp. 1962-1966, 1970.

[27] M. M. Pauthenier and M. Moreau-Hanot, "Charging of Spherical Particles in an Ionising Field", J. Phys. Rad., Vol. 3, pp. 590-613, 1932.

[28] A. Putnam, "Integratable Form of the Drop Drag Co-Efficient", J. Am. Rocket Sci. Vol. 31, pp. 1467-1468, 1961.

[29] J. S. Shrimpton, A. J. Yule, A. P. Watkins, W. Balachandran and D. Hu, "Electrostatically Atomized Hydrocarbon Sprays", Fuel J., Vol. 74, pp. 1094-1103, 1995.

[30] J. S. Shrimpton and A. J. Yule, "Electrohydrodynamics of Charge Injection Atomization : Regimes and Fundamental Limits", Atomization and Sprays, Vol. 13, pp. 173-190, 2002.

[31] J. S. Shrimpton and A. J. Yule, "Design Issues Concerning Charge Injection Atomizers, Accepted for Publication, Atomization and Sprays, 2003.

[32] Y. Hardalupas, A. M. K. P. Taylor, and J. H. Whitelaw, "Phase Validation Criteria of Size Measurements for the Phase Doppler Technique", Expt. Fluids, Vol. 17, pp. 253-258, 1994.

[33] A. K. M. P. Taylor, "Optically Based Measurement Techniques for Dispersed Two Phase Flows", in 'Combusting Flow Diagnostics', pp. 233-289, Durao et al. (eds), Pub. Kluwer, 1992.

John Shrimpton gained his Batchelor degree from Birmingham University (UK) in 1991 and the Ph.D. degree from UMIST (UK) in 1995. After post doctoral work in the field of liquid atomization he was awarded a Lectureship at Imperial College London in 1998 and has recently gained (September 2003) an EPSRC advanced fellowship.

Martin Wong graduated from Imperial College London in 2001. 